\title{
Dickkopf-1 in human oral cancer
}

\author{
KENJI OGOSHI ${ }^{1}$, ATSUSHI KASAMATSU ${ }^{1,2}$, MANABU IYODA $^{1}$, KENTARO SAKUMA $^{1}$, \\ MASANOBU YAMATOJI $^{1}$, YOSUKE SAKAMOTO ${ }^{2}$, KATSUNORI OGAWARA $^{2}$, \\ MASASHI SHIIBA $^{2}$, HIDEKI TANZAWA ${ }^{1,2}$ and KATSUHIRO UZAWA ${ }^{1,2}$ \\ ${ }^{1}$ Department of Clinical Molecular Biology, Graduate School of Medicine, Chiba University; ${ }^{2}$ Division of Dentistry \\ and Oral-Maxillofacial Surgery, Chiba University Hospital, 1-8-1 Inohana, Chuo-ku, Chiba 260-8670, Japan
}

Received February 24, 2011; Accepted April 20, 2011

DOI: 10.3892/ijo.2011.1046

\begin{abstract}
Dickkopf-1 (Dkk1), a negative regulator of the Wnt signaling pathway, is implicated in tumorigenesis in several types of cancer. The purpose of this study was to determine the involvement of Dkk1 in oral squamous cell carcinoma (OSCC). We found that Dkk1 is frequently upregulated in OSCC-derived cell lines and primary OSCCs compared with normal counterparts. Unexpectedly, Dkk1-positive cases were correlated significantly $(\mathrm{P}<0.05)$ with a low risk of regional lymph node metastasis. We also found that cellular migration and invasiveness increased in Dkk1 knockdown cells and decreased in Dkk1 overexpressed cells. Furthermore, we investigated the relationship between the expression of Dkk1 and distribution of $\beta$-catenin in OSCC cells, since the Wnt signaling pathway is related closely to $\beta$-catenin. Whereas alteration of the $\beta$-catenin levels was not observed in each subcellular fractionation, the phosphorylated $\beta$-catenin levels in nuclei increased in Dkk1 knockdown cells and decreased in Dkk1 overexpressed cells. These data indicated that the high phosphorylation level of $\beta$-catenin in nuclei was correlated with a high risk of tumor invasiveness. The current study suggested that Dkk1 plays an important role in regulating cellular migration and invasiveness, making Dkk1 a potential biomarker for early detection of lymph node metastasis in OSCCs.
\end{abstract}

\section{Introduction}

The human Dkk-related gene family is comprised of $D k k l$, $D k k 2, D k k 3$ and $D k k 4$, which contain two distinct cysteinerich domains in which the positions of 10 cysteine residues are highly conserved between family members (1). Dkk1 encodes a secreted protein, which plays a role in head formation in vertebral development, appropriate positioning and development

Correspondence to: Dr Katsuhiro Uzawa, Department of Clinical Molecular Biology, Graduate School of Medicine, Chiba University, Chiba 260-8670, Japan

E-mail: uzawak@faculty.chiba-u.jp

Key words: Dickkopf-1, oral squamous cell carcinoma, migration, invasiveness, phosphorylated- $\beta$-catenin of other organ structures, and is known as a potent inhibitor of the Wnt signaling pathway (2-5). Dkk1 is overexpressed in several cancer types, i.e., breast cancer, Wilms' tumors and multiple myeloma (6-13). In contrast, González-Sancho et al, reported that Dkk1 expression decreased in colon cancer, suggesting that colon carcinogenesis involves removal of the inhibitory effect of Dkk1 on the Wnt signaling pathway.

Wnt is thought to bind both frizzled protein and the low-density lipoprotein receptor-related protein, LRP 5/6, to form a functional ligand-receptor complex that activates the canonical Wnt signaling pathway (14-17). Because $\beta$-catenin plays a crucial role in tumor cells $(18,19)$, the Wnt signaling pathway has been implicated in pathogenesis and progression of human malignancies. With activation of the Wnt signaling pathway, $\beta$-catenin accumulates in the cytoplasm and is translocated into the nucleus and combined with lymphocyte enhancer factor/T-cell factors to activate downstream target genes involved in cancer development $(18,19)$. Previous studies have suggested that high levels of nuclear phosphorylated $\beta$-catenin are associated with low survival rates and possibly related to overexpression of several molecules, such as UPAR, bcl-2 and TIMP-1 (20-23).

In the current study, we analyzed the correlation between Dkk1 expression and the clinicopathological behaviors in oral squamous cell carcinomas (OSCCs) and report that higher Dkk1 expression was associated with a low risk of lymph node metastasis. In addition, Dkk1 knockdown and overexpressed cells showed that Dkk1 regulated the cellular migration and invasiveness in OSCC. We propose that Dkk1 may be a molecular marker for lymph node metastasis in OSCCs.

\section{Materials and methods}

OSCC-derived cell lines and tissue specimens. HSC-2, HSC-3, HSC-4 and SaS, derived from human OSCCs, were purchased from the Human Science Research Resources Bank, Osaka, Japan. H1 and Sa3 were kindly provided by Dr S. Fujita at Wakayama Medical University, Wakayama, Japan. Primary cultured human normal oral keratinocytes (HNOKs) were used as a normal control (24-27). All cells were grown in Dulbecco's modified Eagle's medium (DMEM) (Sigma, St. Louis, MO) supplemented with $10 \%$ fetal bovine serum (FBS) (Sigma) and 50 units $/ \mathrm{ml}$ penicillin and streptomycin (Sigma). 
Primary OSCCs and patient-matched normal oral epithelial samples were obtained during surgical resection at Chiba University Hospital after all patients provided informed consent according to the protocol approved by the institutional review board of Chiba University. The resected tissues were divided into two parts, one of which was frozen immediately and stored at $-80^{\circ} \mathrm{C}$ until RNA isolation, and the second of which was fixed in $10 \%$ buffered formaldehyde solution for pathologic diagnosis and immunohistochemistry (IHC). Histopathological diagnosis of each tissue was performed according to the World Health Organization criteria by the Department of Pathology, Chiba University Hospital. Clinicopathological staging was determined according to the tumor-nodemetastases classification of the International Union against Cancer.

Preparation of cDNA. Total RNA was isolated using TRIzol Reagent (Invitrogen, Carlsbad, CA) according to the manufacturer's instructions. cDNA was generated from $5 \mu \mathrm{g}$ of total RNA using Ready-To-Go You-Prime First-Strand Beads (GE Healthcare, Buckinghamshire, UK) and oligo(dT) primer (Sigma Genosys, Ishikari, Japan) according to the manufacturer's instructions.

mRNA expression analysis. Real-time quantitative reverse transcriptase-polymerase chain reaction (qRT-PCR) was performed to evaluate the expression level of $D k k l \mathrm{mRNA}$ in the OSCC-derived cell lines (HSC-2, HSC-3, HSC-4, Sa3, $\mathrm{SaS}$ and $\mathrm{H1}$ ) and HNOKs. Primary OSCCs and paired specimens of normal oral tissues obtained from 96 patients also were evaluated. qRT-PCR was performed with a single method using a LightCycler FastStart DNA Master SYBR-Green I Kit (Roche Diagnostics GmbH, Mannheim, Germany). The nucleotide sequences of gene-specific primers for qRT-PCR amplification were as follows: $D k k l$ forward 5'-CAGGCTTGC AAAGTGACGGT-3' and reverse 5'-TACCATCGCGACAAA GACCC-3'. Amplified products were analyzed by $3 \%$ agarose gel electrophoresis to ascertain size and purity. The PCR reactions using the LightCycler apparatus were carried out in a final volume of $20 \mu \mathrm{l}$ of a reaction mixture comprised of $2 \mu \mathrm{l}$ of FirstStart DNA Master SYBR-Green I mix (Roche), $3 \mathrm{mM}$ of $\mathrm{MgCl}_{2}$, and $1 \mu \mathrm{M}$ of the primers, according to the manufacturer's instructions. The reaction mixture then was loaded into glass capillary tubes and subjected to an initial denaturation at $95^{\circ} \mathrm{C}$ for $10 \mathrm{~min}$, followed by 45 rounds of amplification at $95^{\circ} \mathrm{C}(10 \mathrm{sec})$ for denaturation, $68^{\circ} \mathrm{C}(10 \mathrm{sec})$ for annealing, and $72^{\circ} \mathrm{C}(10 \mathrm{sec})$ for extension, with a temperature slope of $20^{\circ} \mathrm{C} / \mathrm{sec}$. The transcript amount for the $D k k l$ gene was estimated from the respective standard curves and normalized to the glyceraldehyde-3-phosphate dehydrogenase (GAPDH) (forward 5'-CATCTCTGCCCCCTCTGCTGA-3' and reverse 5'-GGAT GACCTTGCCCACAGCCT-3') transcript amount determined in corresponding samples.

Protein extraction. The cells were washed twice with cold phosphate buffered saline (PBS) and centrifuged at $500 \mathrm{x} \mathrm{g}$. Cytoplasmic and nuclear fractions from cultured cells were isolated using the NE-PER Nuclear and Cytoplasmic Extraction Reagents (Thermo, Rockford, IL). The protein concentration was measured using the BCA Protein Assay Kit (Thermo).
Western blot analysis. Cytoplasmic and nuclear proteins $(50 \mu \mathrm{g})$ were separated by sodium dodecyl sulfate polyacrylamide gel electrophoresis in 4-12\% gel, transferred to nitrocellulose membranes, and blocked for $1 \mathrm{~h}$ at room temperature in Blocking One (Nacalai Tesque, Tokyo, Japan). The membranes were incubated with anti-Dkk1 (Santa Cruz Biotechnology, Santa Cruz, CA), $\alpha$-tubulin (Santa Cruz Biotechnology), $\beta$-catenin (Becton-Dickinson, Franklin Lakes, NJ) or phosphorylated ser33/ser37/thr41 $\beta$-catenin (p- $\beta$-catenin; Cell Signaling Technology Japan, Tokyo, Japan) antibodies for $4 \mathrm{~h}$ at room temperature. The membranes were washed with $0.1 \%$ Tween- 20 in Tris-buffered saline and incubated with secondary antibodies, coupled to horseradish peroxidase-conjugated anti-rabbit or anti-mouse IgG, in Blocking One for $1 \mathrm{~h}$ at room temperature. The proteins were detected by SuperSignal Chemiluminescent substrate (Thermo). Finally, immunoblotting results were visualized by exposing the membrane to a cooled CCD camera system (Light Capture, ATTO, Tokyo, Japan). Signal intensities were quantitated using the CS Analyzer version 3.0 (ATTO).

IHC. IHC was performed on 4- $\mu \mathrm{m}$ sections of paraffinembedded specimens using anti-Dkk1 polyclonal antibody (Santa Cruz Biotechnology). Briefly, after deparaffinization and hydration, the endogenous peroxidase activity was quenched by 30 -min incubation in a mixture of $0.3 \%$ hydrogen peroxide solution in $100 \%$ methanol; the sections were blocked for $2 \mathrm{~h}$ at room temperature with 1.5\% blocking serum (Santa Cruz Biotechnology) in PBS before reacting with anti-Dkk1 antibody (1:100 dilution) at $4^{\circ} \mathrm{C}$ in a moist chamber overnight. Upon incubation with the primary antibody, the specimens were washed three times in PBS and treated with Histofine Simplestain Max-PO (G) (Nichirei, Tokyo, Japan) followed by color development in 3,3'-diaminobenzidine tetrahydrochloride (Dako, Carpinteria, CA). Finally, the slides were counterstained lightly with hematoxylin, dehydrated with ethanol, cleaned with xylene and mounted. Non-specific binding of an antibody to proteins other than the antigen sometimes occurred. To investigate non-specific binding of the Dkk1 antibody, an immunizing peptide blocking experiment was performed. As a negative control, triplicate sections were immunostained without exposure to primary antibodies, thus confirming the staining specificity. To quantify the state of Dkk1 protein expression in those components, we used IHC score systems described previously $(26,28)$. The intensity of the Dkk1 immunoreaction was scored as follows: $1+$, weak; $2+$, moderate; and $3+$, intense. The cell number and the staining intensity then were multiplied to produce a Dkk1 IHC score. Cases with a Dkk1 IHC score exceeding 85.3 (the highest score for normal tissue) were defined as Dkk1-positive. Two independent pathologists, neither of whom had any knowledge of the patients' clinical status, made these judgments.

Transfection of Dkk1 shRNA and overexpressed plasmids. OSCC-derived cells (Sa3 and H1) were transfected with Dkk1 shRNA (shDkk1, Santa Cruz Biotechnology), control shRNA (shMock, Santa Cruz Biotechnology), overexpressed Dkk1 (oeDkk1, Myc-tagged, OriGene Technologies, Rockville, MD), and control vector (oeMock, Myc-tagged, OriGene Technologies) using Lipofectamine LTX (Invitrogen). The NIH3T3 cell line 
A

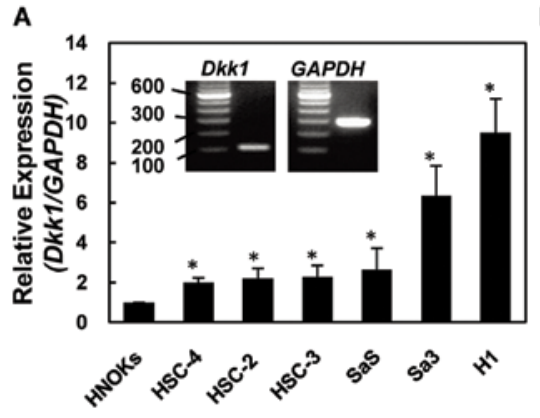

C
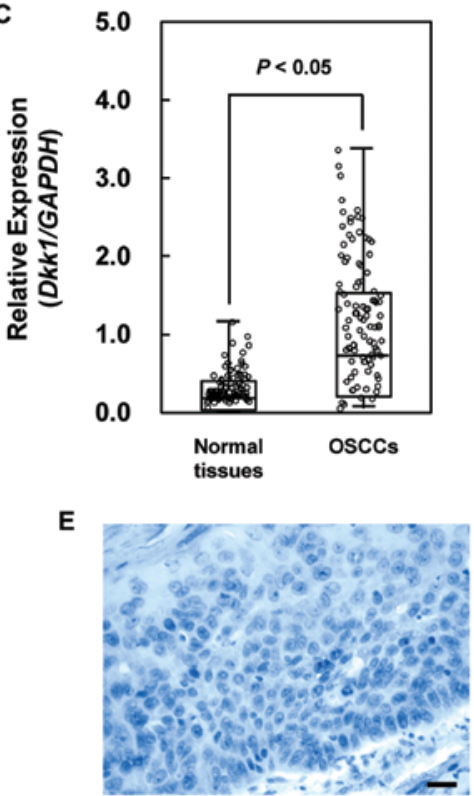

B

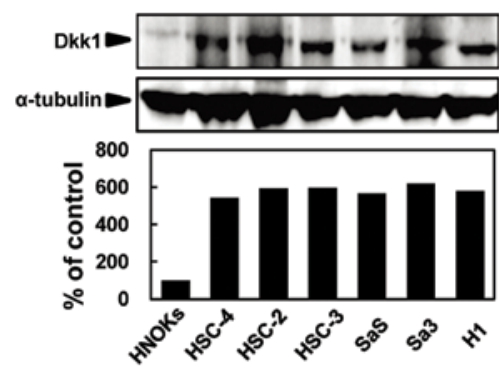

D
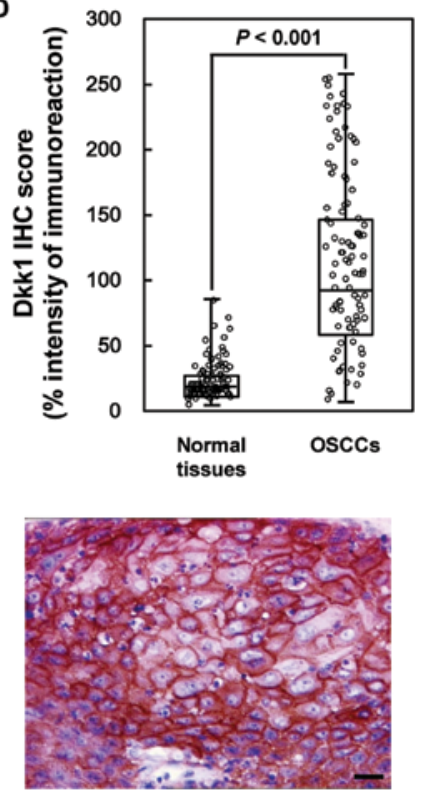

Figure 1. Evaluation of Dkk1 expression in OSCC-derived cell lines and primary OSCCs. (A) Dkk1 mRNA levels were analyzed in OSCC-derived cells and HNOKs by qRT-PCR analysis. Significant up-regulation of Dkk1 mRNA is seen in six OSCC-derived cell lines compared with that in HNOKs ("P<0.05; Mann-Whitney U test). Data are expressed as the means \pm SEM in triplicate. The PCR product is electrophoresed on 3\% agarose gel and visualized using ethidium bromide stain. The band appears as the appropriate size. (B) Representative Western blot data of Dkk1 in OSCC-derived cell lines and HNOKs. The densitometric data of Western blot analysis of Dkk1. Dkk1 protein is up-regulated in OSCC-derived cell lines compared with that in HNOKs. (C) The relative mRNA expression levels in normal oral tissues $(\mathrm{n}=96)$ and matched primary OSCCs $(\mathrm{n}=96)$ range from 0.001 to 1.146 (median, 0.139$)$ and 0.051 to 3.387 (median, 0.648), respectively. $D k k 1$ expression in primary OSCCs is significantly ( $\mathrm{P}<0.05$; Mann-Whitney $\mathrm{U}$ test) higher than in matched normal oral tissues. Data are expressed as the means \pm SEM of three independent experiments. (D) The state of Dkk1 protein expression in normal oral tissues (n=96) and primary OSCCs $(\mathrm{n}=96)$. The Dkk1 IHC scores are calculated as follows: IHC score $=1 \mathrm{x}$ (no. of weakly stained cells in the field) $+2 \mathrm{x}$ (no. of moderately stained cells in the field) $+3 \times$ (no. of intensely stained cells in the field). The Dkk1 IHC scores for normal oral tissues and OSCCs ranged from 4.23 to 85.25 (median, $14.89)$ and 7.41 to 258.43 (median, 96.35), respectively. Dkk1 protein expression levels in OSCCs are significantly ( $<<0.001$; Mann-Whitney U test) higher than that in normal oral tissues. (E and F) Representative IHC results of Dkk1 in normal oral tissue and primary OSCC. (E) Normal oral tissue exhibited negative Dkk1 protein expression. Original magnification, $\mathrm{x} 400$. Scale bars, $10 \mu \mathrm{m}$. (F) Positive immunoreactivity for Dkk1 in OSCCs is detected in the cytoplasm. Original magnification, $\mathrm{x} 400$. Scale bars, $10 \mu \mathrm{m}$.

also was transfected with oeDkk1 or oeMock vectors. After transfection, the cells stably expressing shDkk1 and oeDkk1 were isolated using puromycin (Santa Cruz Biotechnology) and neomycin (Invitrogen), respectively. Two to 3 weeks after transfection, viable colonies were picked up and transferred to new dishes.

Cellular growth. To evaluate the effect of Dkk1 knockdown on cellular growth, we performed a cellular proliferation assay. The experiments were carried out for $168 \mathrm{~h}$ by counting the numbers of cells every $24 \mathrm{~h}$. The cells in three samples were trypsinized and counted using a hemocytometer at the indicated time-point.

Migration assay. To evaluate the effect of Dkk1 knockdown on migration, we performed the wound healing assay described previously (29). Briefly, after uniform wounds were made in confluent culture of shDkk1 and shMock cells, the extent of closure was monitored visually every $4 \mathrm{~h}$ for $24 \mathrm{~h}$. The results were visualized by measuring the wound spaces using the Lenalaf220 software (http://www.vector.co.jp/download/file/ win95/art/fh442375.html). The mean value was calculated from data obtained from three separate chambers.

Invasiveness assay. To evaluated the effect of Dkk1 knockdown and Dkk1 overexpression on invasiveness, we performed an invasiveness assay. A total of $2.5 \times 10^{5}$ cells were seeded on a polyethylene terephthalate membrane insert with a pore size of $3 \mu \mathrm{m}$ in a transwell apparatus (Becton-Dickinson). In the lower chamber, $1 \mathrm{ml}$ of DMEM with $10 \%$ FBS and puromycin or neomycin was added as a chemoattractant. After the cells were incubated for $22 \mathrm{~h}$ at $37^{\circ} \mathrm{C}$, the inset was washed with PBS, 
Table I. Correlation between Dkk1 expression and parameters in OSCCs.

Results of immunostaining

No. of patients

\begin{tabular}{|c|c|c|c|c|}
\hline Parameter & Total & $\operatorname{Dkk1}(-)(\%)$ & $\operatorname{Dkk1}(+)(\%)$ & P-value \\
\hline \multicolumn{5}{|l|}{ Age at surgery } \\
\hline$<60$ & 26 & $13(50)$ & $13(50)$ & \multirow[t]{3}{*}{0.97} \\
\hline $60-70$ & 22 & 7 (32) & $15(68)$ & \\
\hline$>70$ & 48 & $22(46)$ & $26(54)$ & \\
\hline \multicolumn{5}{|l|}{ Gender } \\
\hline Male & 58 & $26(45)$ & $32(55)$ & \multirow[t]{2}{*}{0.94} \\
\hline Female & 38 & $16(42)$ & $22(22)$ & \\
\hline \multicolumn{5}{|l|}{ T-primary tumor size } \\
\hline $\mathrm{T} 1$ & 8 & 1 (13) & $7(87)$ & \multirow[t]{4}{*}{$0.040^{\mathrm{a}}$} \\
\hline $\mathrm{T} 2$ & 63 & 27 (43) & $36(57)$ & \\
\hline T3 & 14 & $7(50)$ & $7(50)$ & \\
\hline $\mathrm{T} 4$ & 11 & 7 (64) & $4(36)$ & \\
\hline \multicolumn{5}{|l|}{ N-regional lymph node } \\
\hline Negative & 59 & $21(36)$ & $38(64)$ & \multirow[t]{2}{*}{$0.043^{\mathrm{a}}$} \\
\hline Positive & 37 & $21(56)$ & $16(44)$ & \\
\hline \multicolumn{5}{|l|}{ TNM stage } \\
\hline I & 7 & 1 (14) & $6(86)$ & \multirow[t]{4}{*}{$0.028^{\mathrm{a}}$} \\
\hline II & 43 & $17(40)$ & $26(60)$ & \\
\hline III & 16 & $6(38)$ & $10(62)$ & \\
\hline IV & 30 & $18(60)$ & $12(40)$ & \\
\hline \multicolumn{5}{|l|}{ Histopathological type } \\
\hline Well-differentiated & 61 & 24 (39) & $37(61)$ & \multirow[t]{3}{*}{0.18} \\
\hline Moderately differentiated & 30 & $14(47)$ & $16(53)$ & \\
\hline Poorly differentiated & 5 & $4(80)$ & $1(20)$ & \\
\hline \multicolumn{5}{|l|}{ Tumor site } \\
\hline Gingiva & 25 & $9(36)$ & $16(64)$ & \multirow[t]{6}{*}{0.54} \\
\hline Tongue & 52 & $25(25)$ & $27(52)$ & \\
\hline Buccal mucosa & 10 & $3(30)$ & $7(70)$ & \\
\hline Oral floor & 6 & $3(50)$ & $3(50)$ & \\
\hline Oropharyngeal isthmus & 1 & $1(100)$ & $0 \quad(0)$ & \\
\hline Soft palate & 2 & $1(50)$ & $1(50)$ & \\
\hline Total & 96 & 42 (44) & $54(56)$ & \\
\hline
\end{tabular}

Dkk1(-), down-regulated Dkk1; Dkk1(+), up-regulated Dkk1. ${ }^{\mathrm{a} P}<0.05$.

and cells on the top surface of the insert were removed with a cotton swab. Cells adhering to the lower surface of the membrane were fixed with methanol and stained with crystal violet; the numbers of cells invading the pores in five random fields were counted using a light microscope at x100 magnification.

\section{Results}

Evaluation of Dkkl expression in OSCC-derived cell lines. We examined $D k k 1 \mathrm{mRNA}$ and protein expression in six OSCC-derived cell lines (HSC-2, HSC-3, HSC-4, Sa3, SaS and H1) and HNOKs using qRT-PCR and Western blot analyses. Significant up-regulation of $D k k 1$ mRNA occurred in all OSCC cell lines compared with the HNOKs (Fig. 1A)
( $\mathrm{P}<0.05)$. The PCR product was electrophoresed on 3\% agarose gel and visualized using ethidium bromide stain. Appropriate bands were observed (Dkk1, 109 bp; GAPDH, 316 bp) (Fig. 1A). Fig. 1B shows representative results of the Western blot analysis. The molecular weight of Dkk1 was $35 \mathrm{kDa}$. The values obtained from densitometric analysis of Dkk1 protein were normalized to $\alpha$-tubulin levels and then expressed as a percentage of the HNOK values. A significant increase in Dkk1 protein expression was seen in all OSCC cell lines compared with the HNOKs.

Evaluation of Dkkl expression in primary OSCCs. We evaluated Dkkl mRNA expression in the normal oral tissues and paired primary OSCCs from 96 patients. Similar to the data obtained from the OSCC-derived cell lines, qRT-PCR showed that Dkkl 

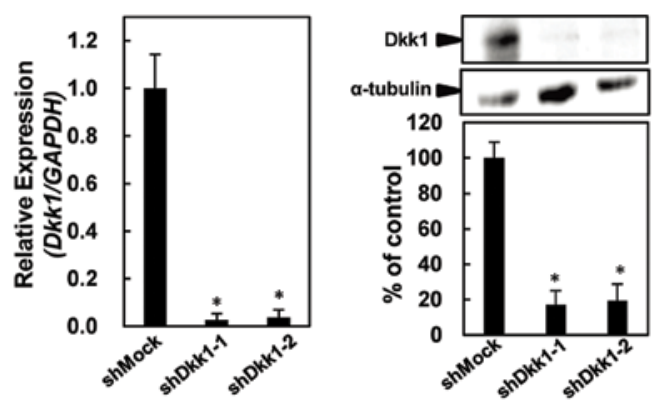

D
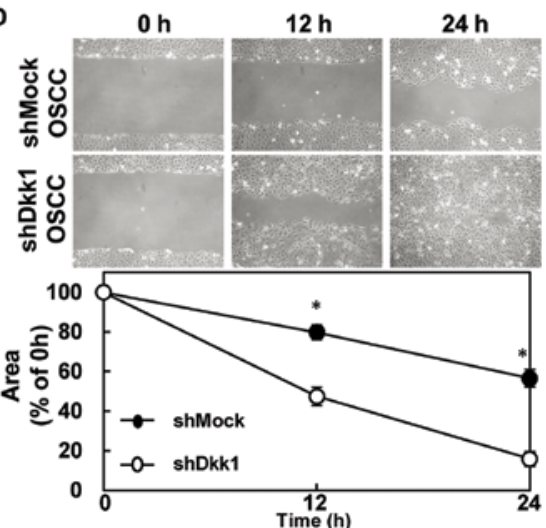

C

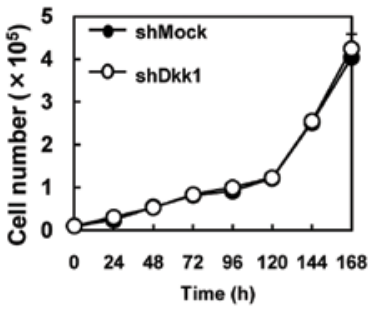

E
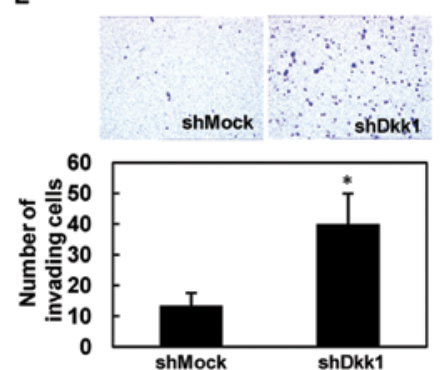

Figure 2. Functional analyses of Dkk1 knockdown cells. Significant down-regulation of $D k k 1$ mRNA (A) and protein (B) is seen in shDkk1 transfected cells compared with that in shMock cells using qRT-PCR and Western blot analyses ( $\mathrm{P}<0.05$; Mann-Whitney U test). (C) Comparison of cellular growth between shDkk1 and shMock cells. Similar growth curves are seen in both transfectants. (D) Wound healing assay of shDkk1 cells. The wound area has decreased significantly in the culture of shDkk1 cells after $24 \mathrm{~h}$, whereas there is still a gap in shMock cells (*P<0.01; Mann-Whitney $\mathrm{U}$ test). (E) Invasiveness assay of shDkk1 cells. After crystal violet staining, the number of cells invading the pores are counted (x100 magnification). The numbers of penetrating shDkk1 cells (40.04 \pm 9.89$)$ are significantly ( $\mathrm{P}<0.01$; Mann-Whitney U test) greater compared to shMock cells $(13.40 \pm 4.16)$.

mRNA expression was up-regulated in $76(80 \%)$ of 96 primary OSCCs compared with the matched normal oral tissues. The relative mRNA expression levels in the normal oral tissues and primary OSCCs ranged from 0.001 to 1.146 (median, 0.139) and 0.051 to 3.387 (median, 0.648), respectively (Fig. 1C) $(\mathrm{P}<0.05)$.

In addition to the $D k k 1 \mathrm{mRNA}$ expression data in primary OSCCs, Dkk1 protein expression in primary OSCC was significantly $(\mathrm{P}<0.001)$ higher than in normal tissues (Fig. 1D). The Dkk1 IHC scores of the normal oral tissues and primary OSCCs ranged from 4.23 to 85.25 (median, 14.89) and 7.41 to 258.43 (median, 96.35), respectively. Representative IHC results for Dkk1 staining in normal oral tissue and primary OSCC are shown in Fig. 1E and F, respectively. The correlation between the clinicopathological characteristics of patients with OSCC and the status of Dkk1 protein expression using IHC score system are shown in Table I. Dkk1-positive OSCCs were correlated with regional lymph node metastasis $(\mathrm{P}=0.043)$, tumor size $(\mathrm{P}=0.040)$ and TMN stage $(\mathrm{P}=0.028)$.

Functional analyses of Dkk1 knockdown cells. Since Dkk1positive cases were associated with OSCC without lymph node metastasis, we assumed that Dkk1 might regulate cellular migration and invasiveness in OSCC. To obtain stable Dkk1 knockdown cells, we transfected the shDkk1 and shMock plasmids into OSCC-derived cells. The qRT-PCR data showed that the $D k k 1$ mRNA expression in shDkk1 cells was significantly ( $\mathrm{P}<0.05)$ lower than in shMock cells (Fig. 2A). Dkk1
A

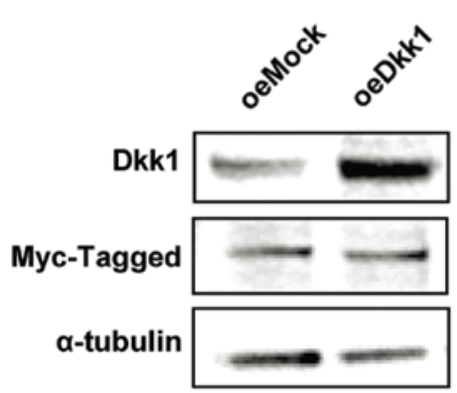

B

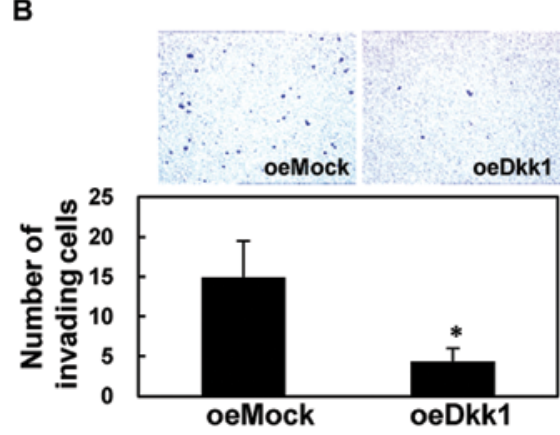

Figure 3. Invasiveness-related biology in Dkk1 overexpressed cells. (A) Significant up-regulation of Dkk1 protein is seen in Dkk1 overexpressed cells (oeDkk1) compared with that in mock transfected cells (oeMock) using Western blot analyses. (B) Invasiveness assay of oeDkk1 cells. After crystal violet staining, the numbers of cells entering the pores are counted (x100 magnification). The numbers of penetrating oeDkk1 cells $(4.33 \pm 1.67)$ are significantly ("P $<0.05$; Mann-Whitney U test) fewer compared with oeMock cells $(14.91 \pm 4.56)$. 
A

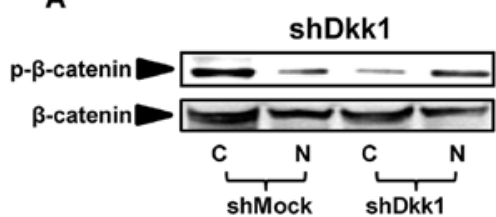

C

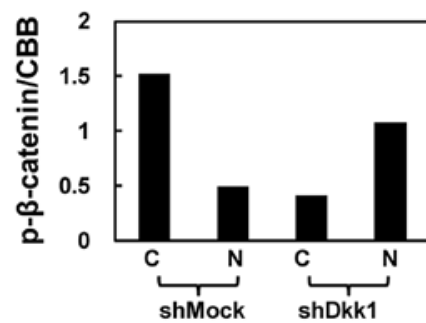

E

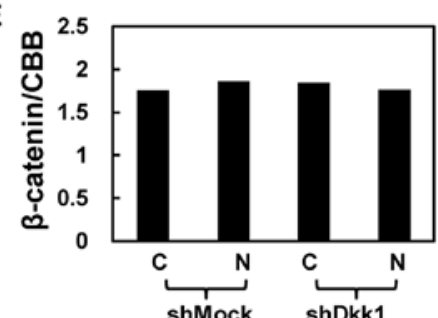

$\mathbf{G}$

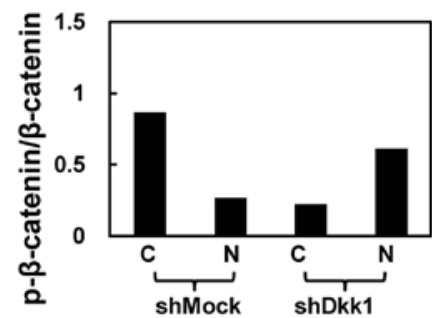

B

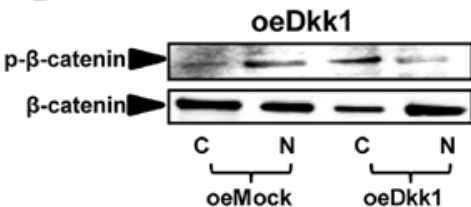

D

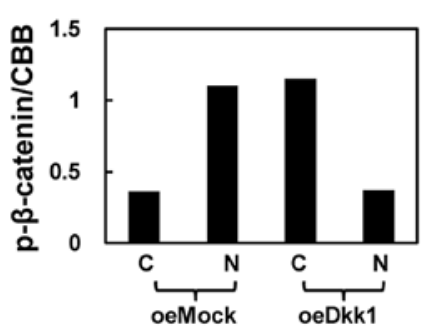

$\mathbf{F}$

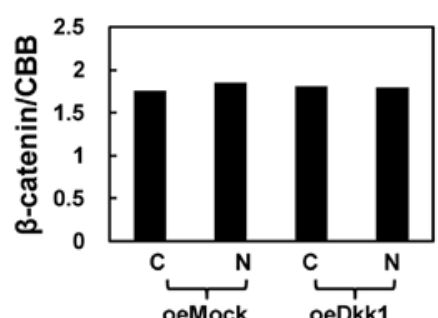

H

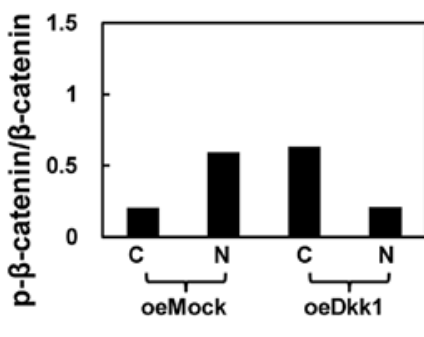

Figure 4. Phosphorylated $\beta$-catenin levels in shDkk1 and oeDkk1 cells. In Dkk1 knockdown and overexpressed cells, $\beta$-catenin expression does not differ between the cytoplasm and nucleus; translocation of $\beta$-catenin from the cytoplasm to the nuclei correlated Dkk1 expression is not significant (A, B, E and F). In shDkk1 cells, the phosphorylation ratio of $\beta$-catenin (p- $\beta$-catenin/ $\beta$-catenin) in the nuclei increases with decreasing Dkk1 expression (A, $C$, E and $G)$. In oeDkk1 cells, p- $\beta$-catenin/ $\beta$-catenin in the nuclei decreases with increasing Dkk1 expression $(\mathrm{B}, \mathrm{D}, \mathrm{F}$ and $\mathrm{H})$. The values obtained from densitometric analysis of each protein were normalized to protein stained with Coomassie Brilliant Blue (CBB).

protein levels in shDkk1 cells also decreased significantly $\left({ }^{*} \mathrm{P}<0.05\right)$ compared with shMock cells by Western blot analysis (Fig. 2B). To evaluate the effect of Dkk1 knockdown on cellular growth, we performed a cellular proliferation assay. Similar growth curves of both transfectants were seen, indicating that down-regulation of Dkk1 did not affect cellular proliferation (Fig. 2C). We then performed cellular migration and invasiveness assays to determine the biologic effects in Dkk1 knockdown cells. In a migration assay, when we visually monitored the area of uniform wounds in confluent cell culture, the wounds in Dkk1 knockdown cells closed earlier than in shMock cells (Fig. 2D). In the invasiveness assay, the number of penetrating Dkk1 knockdown cells increased compared with shMock cells (Fig. 2E). Therefore, Dkk1 knockdown cells showed increased migration and invasiveness activities.

Invasiveness-related biology in Dkkl overexpressed cells. Using Western blot analysis, we established that Dkk1 overexpressed OSCC-derived cells (oeDkk1 cells) and confirmed up-regulation of Dkk1 in oeDkk1 cells compared with oeMock cells (Fig. 3A).
The invasiveness assay using oeDkk1 cells was performed to investigate the biologic effect of exogenous Dkk1. The oeDkk1 cells showed decreased invasiveness compared with oeMock cells (Fig. 3B).

Phosphorylated $\beta$-catenin levels in Dkkl knockdown and overexpressed cells. Using Western blot analysis, we investigated the phosphorylated $\beta$-catenin levels in nuclei using shDkk1 and oeDkk1 cells. $\beta$-catenin expression did not differ in the nuclei and cytoplasm in Dkk1 knockdown and overexpressed cells (Fig. 4A, B, E and F). The phosphorylation ratio of $\beta$-catenin ( $p$ - $\beta$-catenin $/ \beta$-catenin) in the nuclei of shDkk1 cells increased compared with shMock cells (Fig. 4A, $\mathrm{C}, \mathrm{E}$ and $\mathrm{G})$. $\mathrm{p}$ - $\beta$-catenin/ $\beta$-catenin in the nuclei of oeDkk1 cells also decreased compared with oeMock cells (Fig. 4B, D, $\mathrm{F}$ and $\mathrm{H})$.

Invasiveness-related biology and phosphorylated $\beta$-catenin level in Dkkl-overexpressed NIH3T3 cells. We established the Dkk1-overexpressed NIH3T3 cells (oeDkk1-NIH3T3 cells) and 
then confirmed up-regulation of Dkk1 in the oeDkk1-NIH3T3 cells compared with oeMock-NIH3T3 cells by Western blot analysis. An invasiveness assay of the oeDkk1-NIH3T3 cells was performed to investigate the effect of exogenous human Dkk1. The invasiveness activity in the oeDkk1-NIH3T3 cells significantly increased compared with the oeMock-NIH3T3 cells, which was consistent with a previous study (24) that migration and invasiveness increased in human Dkk1overexpressed mouse fibroblasts. A high phosphorylated $\beta$-catenin level was found in the nuclei of oeDkk1-NIH3T3 cells compared with mock-NIH3T3 cells (data not shown).

\section{Discussion}

We found that $D k k l$ is one of the most significantly up-regulated genes in OSCC, $D k k l$ plays an important role in tumor invasiveness and $D k k l$ could be involved in regulation of phosphorylation of $\beta$-catenin in nuclei. Therefore, our data suggested that Dkk1 controls lymph node metastasis by regulating phosphorylated $\beta$-catenin levels in the nuclei of OSCC.

Only a few studies have reported the clinical or prognostic relevance of Dkk1 in human cancers. The Dkk1 expression level at clinical stage I in endometrial carcinoma was significantly greater than at other stages (30). High Dkk1 expression also was found in the early developmental stage of prostate cancer but not in late-stage bone metastasis, suggesting that Dkk1 expression increases in early-stage cancer and is a necessary early event to support osteolysis (31). Thus, these results were consistent with our observation that higher Dkk1 expression was correlated with low risk of regional lymph node metastasis. The invasiveness of endometrial carcinoma cells, which were treated with exogenous Dkk1 for $24 \mathrm{~h}$, decreased compared with control cells, indicating that invasiveness was suppressed by exogenous Dkk1 (31). Our Dkk1 knockdown and overexpression experiments also showed that Dkk1 regulated tumor invasiveness in OSCC cells.

The Wnt signal pathway plays roles in cancer development and progression through $\beta$-catenin activation (18,32-39). Some studies have shown that phosphorylated $\beta$-catenin was immunodetected in both the cytoplasm and nuclei of cancer cells (21) and that phosphorylated $\beta$-catenin expression was localized almost exclusively in the nuclei in cancers, such as melanoma and colorectal cancer $(20,40)$. Nuclear localization of phosphorylated $\beta$-catenin may be related to changes at other stages in the signaling pathway $(20-23,40)$. A high level of nuclear phosphorylated $\beta$-catenin was involved in the increased invasiveness phenotype and in the low survival rates of melanoma and breast cancer $(20,21)$. Because nuclear phosphorylated $\beta$-catenin was implicated in the aggressiveness and invasiveness of tumor cells through its positive correlation with invasiveness and antiapoptotic molecules, uPAR, bcl-2, and TIMP-1, nuclear phosphorylated $\beta$-catenin may be a more sensitive probe for biologically significant activation of tumor cells (21-23). The current study suggests that Dkk1 regulates phosphorylated $\beta$-catenin level in nuclei, which would be correlated with the migration and invasiveness of OSCC cells.

In conclusion, Dkk1 is overexpressed frequently in OSCCs and could play important roles in the course of regional lymph node metastasis by regulating migration and invasiveness.
Dkk1 is likely to be not only a molecular marker for early detection of lymph node metastasis but also an efficacious treatment strategy for preventing cancer metastasis in OSCCs.

\section{Acknowledgements}

We thank Lynda C. Charters for editing this manuscript.

\section{References}

1. Fedi P, Bafico A, Soria AN, et al: Isolation and biochemical characterization of the human Dkk-1 homologue, a novel inhibitor of mammalian Wnt signaling. J Biol Chem 274: 19465-19472, 1999.

2. Glinka A, Wu W, Delius H, Monaghan AP, Blumenstock C and Niehrs C: Dickkopf-1 is a member of a new family of secreted proteins and functions in head induction. Nature 391: 357-362, 1998.

3. Kazanskaya O, Glinka A and Niehrs C: The role of Xenopus dickkopf 1 in prechordal plate specification and neural patterning. Development 127: 4981-4992, 2000.

4. Schneider VA and Mercola M: Wnt antagonism initiates cardiogenesis in Xenopus laevis. Genes Dev 15: 304-315, 2001.

5. Niida A, Hiroko T, Kasai M, et al: Dkk1, a negative regulator of Wnt signaling, is a target of the $\beta$-catenin/TCF pathway. Oncogene 23: 8520-8526, 2004.

6. Forget MA, Turcotte S, Beauseigle D, et al: The Wnt pathway regulators Dkk1 is preferentially expressed in hormone-resistant breast tumors and in some common cancer types. $\mathrm{Br} \mathrm{J}$ Cancer 96: 646-653, 2007

7. Yamabuki T, Takano A, Hayama S, et al: Dikkopf-1 as a novel serologic and prognostic biomarker for lung and esophageal carcinomas. Cancer Res 67: 2517-2525, 2007.

8. Wirths $\mathrm{O}$, Waha A, Weggen S, et al: Overexpression of human Dickkopf-1, an antagonist of wingless/WNT signaling, in human hepatoblastomas and Wilms' tumors. Lab Invest 83: 429-434, 2003.

9. Patil MA, Chua MS, Pan KH, et al: An integrated data analysis approach to characterize genes highly expressed in hepatocellular carcinoma. Oncogene 24: 3737-3747, 2005

10. Tian E, Zhan F, Walker R, et al: The role of the Wnt-signaling antagonist DKK1 in the development of osteolytic lesions in multiple myeloma. N Engl J Med 349: 2483-2494, 2003.

11. Gosepath EM, Eckstein N, Hamacher A, et al: Acquired cisplatin resistance in the head-neck cancer cell line Cal27 is associated with decreased DKK1 expression and can partially be reversed by overexpression of DKK1. Int J Cancer 123: 2013-2019, 2008.

12. Chamorro MN, Schwartz DR, Vonica A, Brivanlou AH, Cho KR and Varmus HE: FGF-20 and DKK1 are transcriptional targets of beta-catenin and FGF-20 is implicated in cancer and development. EMBO J 24: 73-84, 2005.

13. González-Sancho JM, Aguilera O, García JM, et al: The Wnt antagonist DICKKOPF-1 gene is a downstream target of betacatenin/TCF and is down-regulated in human colon cancer. Oncogene 24: 1098-1103, 2005.

14. Wehrli M, Dougan ST, Caldwell K, et al: Arrow encodes an LDL-receptor-related protein essential for Wingless signalling. Nature 407: 527-530, 2000.

15. Tamai K, Semenov M, Kato Y, et al: LDL-receptor-related proteins in Wnt signal transduction. Nature 407: 530-535, 2000.

16. Pinson KI, Brennan J, Monkley S, Avery BJ and Skarnes WC: An LDL receptor-related protein mediates Wnt signaling in mice. Nature 407: 535-538, 2000.

17. Mao J, Wang J, Liu B, et al: Low-density lipoprotein receptor related protein- 5 binds to Axin and regulates the canonical Wnt signaling pathway. Mol Cell 7: 801-809, 2001.

18. Crawford HC, Fingleton BM, Rudolph-Owen LA, et al: The metalloproteinase matrilysin is a target of beta-catenin transactivation in intestinal tumors. Oncogene 18: 2883-2891, 1999.

19. Polakis P: Wnt signaling and cancer. Genes Dev 14: 1837-1851, 2000.

20. Kielhorn E, Provost E, Olsen D, et al: Tissue microarray-based analysis shows phospho-beta-catenin expression in malignant melanoma is associated with poor outcome. Int $\mathbf{J}$ Cancer 103: 652-656, 2003 
21. Nakopoulou L, Mylona E, Papadaki I, et al: Study of phosphobeta-catenin subcellular distribution in invasive breast carci-nomas in relation to their phenotype and the clinical outcome. Mod Pathol 19: 556-563, 2006.

22. Bertaux B, Hornebeck W, Eisen AZ and Dubertret L: Growth stimulation of human keratinocytes by tissue inhibitor of metalloproteinases. J Invest Dermatol 97: 679-685, 1991.

23. Kirkin V, Joos S and Zörnig M: The role of Bcl-2 family members in tumorigenesis. Biochim Biophys Acta 1644: 229-249, 2004.

24. Kasamatsu A, Uzawa K, Nakashima D, et al: Galectin-9 as a regulator of cellular adhesion in human oral squamous cell carcinoma cell lines. Int J Mol Med 16: 269-273, 2005.

25. Endo Y, Uzawa K, Mochida Y, et al: Sarcoendoplasmic reticulum $\mathrm{Ca}(2+)$ ATPase type 2 down-regulated in human oral squamous cell carcinoma. Int J Cancer 110: 225-231, 2004.

26. Sakuma K, Kasamatsu A, Yamatoji M, et al: Expression status of Zic family member 2 as a prognostic marker for oral squamous cell carcinoma. J Cancer Res Clin Oncol 136: 553-559, 2010.

27. Yamatoji M, Kasamatsu A, Yamano Y, et al: State of homeobox A10 expression as a putative prognostic marker for oral squamous cell carcinoma. Oncol Rep 23: 61-67, 2010.

28. Lombardi DP, Geradts J, Foley JF, Chiao C, Lamb PW and Barrett JC: Loss of KAI1 expression in the progression of colorectal cancer. Cancer Res 59: 5724-5731, 1999.

29. Onda T, Uzawa K, Nakashima D, et al: Lin-7C/VELI3/MALS-3: an essential component in metastasis of human squamous cell carcinoma. Cancer Res 67: 9643-9648, 2007.

30. Yi N, Liao QP, Li T and Xiong Y: Novel expression profiles and invasiveness-related biology function of DKK1 in endometrial carcinoma. Oncol Rep 21: 1421-1427, 2009.
31. Hall CL, Daignault SD, Shah RB, Pienta KJ and Keller ET: Dickkopf-1 expression increase early in prostate cancer development and decreases during progression from primary tumor to metastasis. Prostate 68: 1396-1404, 2008.

32. Logan CY and Nusse R: The Wnt signaling pathway in development and disease. Annu Rev Cell Dev Biol 20: 781-810, 2004.

33. Collavin L and Kirschner MW: The secreted Frizzled-related protein Sizzled functions as a negative feedback regulator of extreme ventral mesoderm. Development 130: 805-816, 2003.

34. Mao B, Wu W, Li Y, et al: LDL-receptor-related protein 6 is a receptor for Dickkopf proteins. Nature 411: 321-325, 2001

35. He TC, Sparks AB, Rago C, et al: Identification of c-MYC as a target of the APC pathway. Science 281: 1509-1512, 1998.

36. Shtutman M, Zhurinsky J, Simcha I, et al: The cyclin D1 gene is a target of the betacatenin/LEF-1 pathway. Proc Natl Acad Sci USA 96: 5522-5527, 1999.

37. Levy L, Neuveut C, Renard CA, et al: Transcriptional activation of interleukin-8 by beta-catenin-Tcf4. J Biol Chem 277: 42386-42393, 2001.

38. Brabletz T, Jung A, Dag S, Hlubek F and Kirchner T: Betacatenin regulates the expression of the matrix metalloproteinase-7 in human colorectal cancer. Am J Pathol 155: 1033-1038, 1999.

39. Huelsken $\mathrm{J}$ and Behrens J: The Wnt signalling pathway. J Cell Sci 115: 3977-3978, 2002.

40. Chung GG, Provost E, Kielhorn EP, Charette LA, Smith BL and Rimm DL: Tissue microarray analysis of beta-catenin in colorectal cancer shows nuclear phospho-beta-catenin is associated with a better prognosis. Clin Cancer Res 7: 4013-4020, 2001. 